\title{
ON THE RELATIONSHIP BETWEEN ENERGY EFFICIENCY AND COMPLEXITY: INSIGHT ON THE CAUSALITY CHAIN
}

\author{
F. RUZZENENTI ${ }^{1} \&$ R. BASOSI ${ }^{1,2}$ \\ ${ }^{1}$ Center for Complex Systems Investigation, University of Siena, Siena, Italy. \\ ${ }^{2}$ Department of Chemistry, University of Siena, Siena, Italy.
}

\begin{abstract}
The relationship between the energy efficiency, energy density and complexity level of the system is here addressed from both thermodynamic and evolutionary perspectives. A case study from economic systems is presented to show that, contrary to widespread opinion, energy efficiency is responsible for energy growth and the complexity leap. This article further examines to what extent complexity, on a historical time scale, may evolve to counterbalance conservative effects brought about by energy efficiency. We analyze structural complexity growth by four different paradigms. An evolutionary pattern is then proposed that may encompass the broad dynamics underlying complexity growth. This evolutionary pattern rests on the hypothesis that thermodynamic evolutionary systems are featured from an ever growing influx of energy driven into the system by self-catalytic processes, which must find its way through the constrains of the system. The system initially disposes of the energy by expanding, in extent and in number of components, up to saturation due to inner or outer constraints. The two counteractive forces, constraints and growing energy flux, expose the systems to new gradients. Every new gradient upon the system represents a symmetry rupture in components' space. By exploring a new gradient, the system imposes further restrictions on its components and increases its overall degree of freedom.

Keywords: complexity, complexity leap, degree of freedom, energy density, energy efficiency, fordian structure, spatial gradients, whole structure.
\end{abstract}

\section{INTRODUCTION}

It is a recent acquisition of economics that energy efficiency may lead to energy growth [1]. The seeming paradox is readily explained by a well-known price mechanism. According to economic literature, energy efficiency lessens the price of energy services and goods. That is to say, as the golden rule predicts, the lower the price, the higher the demand [2]. However, can the demand of energy goods and services grow ad libitum because of an ever-growing energy efficiency? (In literature, this phenomenon is known as "Jevon's paradox" or "rebound effect" [3, 4].) More than a century ago, Stanley Jevons shed new light on the limits, physical (geographical or structural) and economic (market saturation), when he pointed out "we cannot, indeed, always be doubling the length of our railways, the magnitude of our ships, and bridges, and factories" [5].

The dynamic linking energy efficiency and energy consumption (i.e., density energy rate) is much better understood in the field of life sciences. Indeed, there are strong analogies between the energy evolutionary paths of biological and economic systems. Like the paradox of economic development, regarding efficient technologies and energy consumption, organisms or ecosystems displaying more advanced and efficient metabolism are those with a higher energy density rate [6, 7]. Alfred Lotka, at the beginning of the twentieth century, called for a quest for a thermodynamics of life that would create a new ground for the disciplines concerning the biosphere [8].

If the positive relationship between energy efficiency and energy density seems to be a constant of thermodynamic evolutionary systems, the analogy can be extended to a third issue: complexity. It is fundamental knowledge of evolutionary biology that greater complexity is linked to greater efficiency. Evolution has awarded the higher efficiency achieved to the higher structural complexity throughout the system [9]. Furthermore, it is well known that more complexity means higher energy costs and thus, the greater the complexity, the higher the energy density rate. This correlation has to 
be maintained throughout any energy structure. For this reason some scientists have adopted energy density as a unit of complexity [10].

May it be possible that it is the uprising of a higher complexity of structure responsible for offsetting the conservative effect brought about by a more efficient technology or a more efficient organism? In this case, the explanation of the paradox formerly envisaged would rest on the complexity leap of the system following the advent of new technology. Accordingly, the general hypothesis here advanced is that energy efficiency growth has to be considered the cause of complexity leap, ad not vice versa.

Some remarks are needed about the concepts of efficiency and complexity. It is intended here for energy efficiency, an energy conversion process that transforms energy (chemical or thermal) into work. Whilst for complexity, we refer to a feature of the whole structure of the system. Two hierarchical levels are henceforth introduced: the process, converting energy, and the system, consuming energy. (The distinction between "conversion" and "consumption" is somehow misleading and has no physical basis. Yet, when we think of a developed organism, we talk about energy intake and when we discuss its metabolic processes, we refer to a conversion rate (i.e., calories of carbohydrates into calories of ATP).) A new, more efficient process with a higher energy conversion rate may not lead to a lower energy level of the system (consumption), given the same amount of components and extent of the system, if the system modifies its structure in such a way that raises the number or the length of interactions among the components in the unit of time (intensity) or in other words, if the system alters its complexity level.

\section{THE CASE STUDY}

\subsection{Efficiency evolution}

The road freight transport system and the productive structure of the EU were addressed as case study. During the lag of time spanning from the 1970s to the late 1990s, despite a remarkable energy efficiency improvement of transport means, the energy consumption of the whole transport sector increased dramatically. The service demand, expressed in $t \mathrm{~km}$ (tons per kilometer of goods delivered), has undeniably grown for market integration following the birth of the common market and consequently, globalization. Figures nevertheless prove that not only did the average distance of shipments grow, but also the frequency. The road freight transport system became more traffic intensive not just in the $\mathrm{EU}$, but also worldwide. A new, more expansive, more flexible, and more segmented productive sector molded a new transport system and the transport system consequently influenced the productive sector in a similar manner. In the fordian system, factories were uni-located and the productive chain was mainly developed in a single production plant. In the post-fordian system, the productive chain is scattered in many, spaced out plants [11]. This major shift, which featured globalization, is commonly named outsourcing and because it heavily relies on logistics, it boosted transport service demand.

During the two decades, the energy efficiency of trucks displayed a remarkable evolution. We have here considered a series of driving tests, consistent in terms of the road, driving conditions and speed. Tests were developed from the same specialized magazine [12] and concern 111 different European trucks, ranging from 1978 to 1998. Fuel economy (liters of fuel per $100 \mathrm{~km}$ ) improved by about $30 \%$ between this time and adjusted fuel economy (fuel economy divided by the engine power, to account for the power increase) reveals that efficiency actually grew by about 50\% [12].

In Europe, during the same time range, the tkm on the road network increased significantly by $130 \%$ [9]. Such a growth rate cannot be sufficiently explained by either the GDP growth (63\%) or the industrial production (56\%). The modal split shift (all modes grew by 88\%) also fails to explain this 
growth. It is noteworthy that in the EU (and in the rest of the OECD countries as well), the tkm grew more on the side of production means than on the side of final products [13]. In other words, factors markets were more influential than goods markets in driving the demand for transport service [14].

\subsection{Transformation of the productive structure: outsourcing and globalization}

The more suitable indicator to detect the process of outsourcing is the Intra-industry Index $[15,16]$. This indicator measures the value of international trades within the same sector or industry and it is thus determined by the exchanges of raw materials, productive means and semi-products in addition to services integral to the production process. The underlying idea is that an integration of goods markets would lead to a mere growth in the distance traveled and can be regarded as a quantitative change because it that does not affect the structure of the productive system. However, a shift from a fordian to a post-fordian productive structure, which indeed represents a qualitative change, would place its burden mostly on the movement of productive factors.

In the light of this indicator, the major leap in international trade of productive factors occurred in the decade between 1980 and 1990 [17]. This indicator depicts a change in the productive structure that developed throughout the time of globalization. Nevertheless, figures indicate that the peak of this change occurred in the central decade between 1980 and 1990 and this observation seems to confirm the hypothesis that the structural change was anterior to the first energy efficiency leap and not vice versa. In the aftermath of the first oil crisis (1973), the road freight transport system underwent a drastic, worldwide renovation in an attempt to reduce oil-derived fuel consumption. In the USA, the truck engines switched from gasoline to diesel and new speed and weight limits were introduced. In the EU, turbocharged engines and aerodynamics elements were adopted in the heavyduty sector and weight limits were abruptly raised to reduce fuel economy of long-distance vehicles $[12,17]$. The size factor, for example, in Europe was determinant. As early as the mid-1970s, in Europe, many countries raised the weight limits for international transports up to 43.2 tons, nearly doubling the previous average size of vehicles and further improving efficiency of shipments. It is here maintained, therefore, that the energy efficiency change in the road freight transport sector should be regarded as a cause of structural change in economy (globalization, outsourcing and post-fordian) and not as a consequence.

\section{THE COMPLEXITY LEAP}

\subsection{The complexity growth analysis - four paradigms}

We would now like to remark that the productive structure changed in a fashion such as to maximize interactions among components and that such a change should be regarded as a growth in complexity. In accordance with our starting hypothesis that the energy efficiency improvement is counterbalanced by a complexity leap, it is now necessary to show that the structure of the productive system actually became more complex in the shift from a fordian to a post-fordian mode.

It will be stressed out, by means of four paradigms:

- hierarchical levels (centralized control system);

- geographical gradient (scale effect);

- homeostatic circuits (decentralized control system);

- path diversity and length (degree of freedom). 


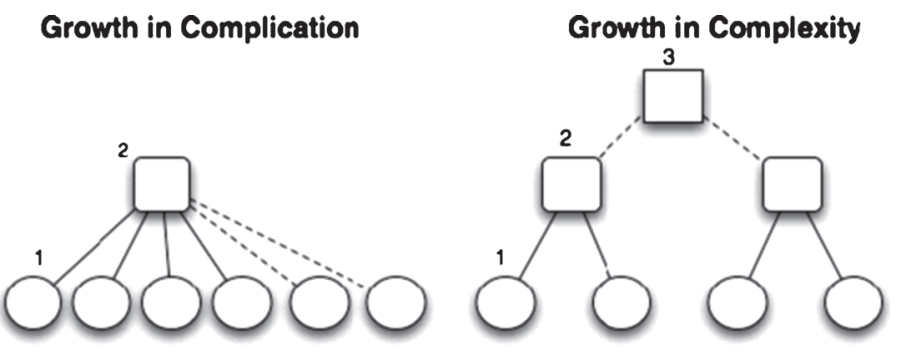

Figure 1: Hierarchical levels (for a centralized control system).
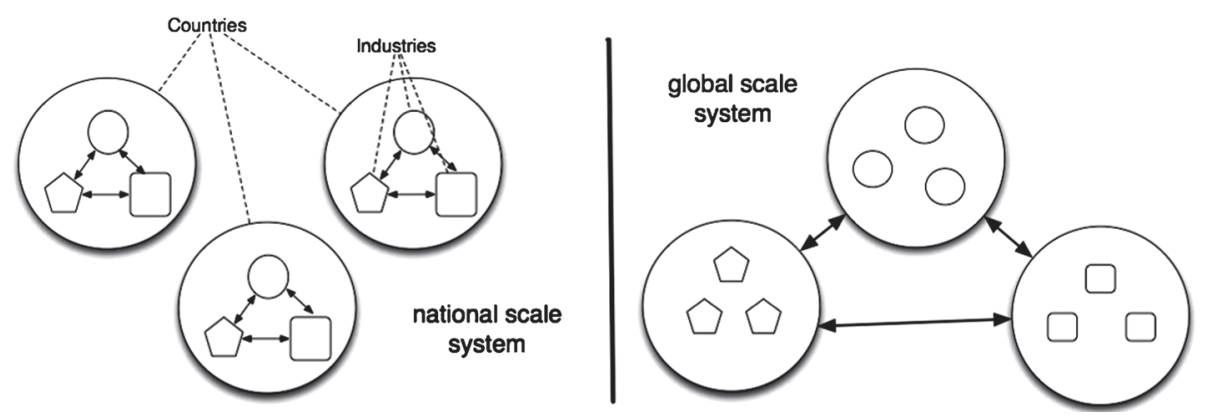

Figure 2: Geographical gradient (scale effect).

Figure 1 depicts hierarchical system asset in which the breakdown of symmetry reduces the degree of freedom for lower level variables. The two graphs (Fig. 1) reveal that the system grew in number of components and connections accounting for the "growth in organization." Only the second graph (on the right), however, illustrates a real growth in organization. The shifting form local to a global productive structure increases the hierarchical chain. Indeed, the system did not grow merely in extent or number of components and a new and higher control level is needed on a global level.

Due to economic scale effects, firms tend to increase in size or productive districts to form. Economic scale effects were fostered by globalization and market integration because more competitive firms could access new, larger markets. Economic scale effects brought the new factor of selection to firms and thus survivors grew and engulfed or displaced minor firms. National specialization can be seen as a geographical gradient in the productive system (Fig. 2). (It is a spatial gradient that relates the diversity of the system to its size and it is an ecological function. In ecology, in fact, biodiversity is also related to the size of the ecosystem [7].) This gradient leads to a breakdown in symmetry, with the rise of a new hierarchical organization level, and to increased average distances of parts (production plants). It will therefore augment both the distance and the frequency of shipments, as already remarked in light of the hierarchical system theory.

A further consequence of scale economy concerns the organization of the production:

1. More specialization and more integration (interdependence) among system components and suppliers and subsidiaries became essential (synchronization).

2. Increase in interactions among system components due to change in the shape of the production chain. 
Fordian siructure<smiles>[O-][O+][O-]</smiles>

Post-ordian structure

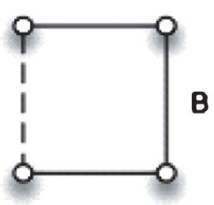

Graph $B$ is more complex than graph A even though it has the same number of points (and lines. in the open loop case) because:

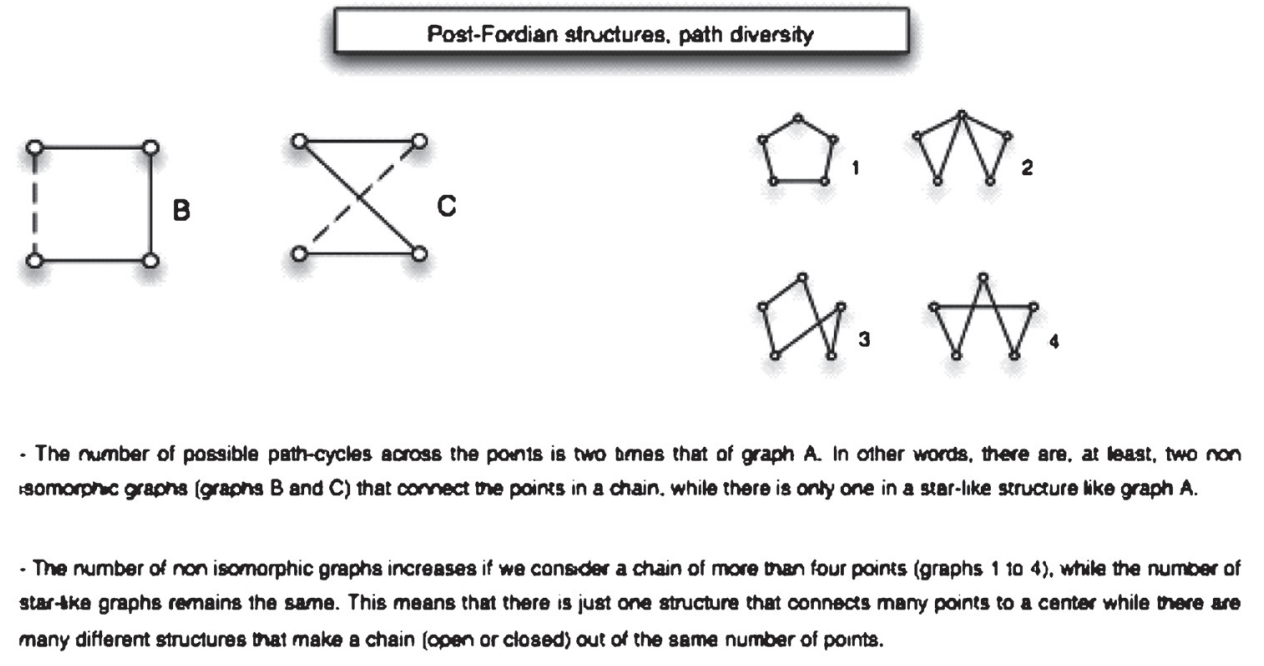

Figure 3: Path diversity and length (degree of freedom).

The first feature mainly concerns the outsourcing process because it affects firms by economically externalizing their functions. For those firms relying on external resources to pursue their productive needs, production becomes less costly, but more subdued due to uncontrollable factors. Part of its activity, formerly controlled managerially and internally, is now focused on free market. This shift reduces the stability of the system and increases its complexity. The homeostatic system theory tells us that economic externalization augmented the complexity because it shifted the control system from a centralized to a decentralized process [18].

In Fig. 3, it is shown by means of graph, the theory that the graph depicting the post-fordian structure is more complex than the one illustrating the fordian structure. In graph A, it is assumed that the productive chain develops thoroughly in a central plan where all the materials converge (star structure). In graphs $\mathrm{B}$ and $\mathrm{C}$, it is assumed that the productive chain develops through various plants scattered on the territory (in an open or closed loop) and the goods collect the materials throughout the productive stages. It is easily proved that the second graph displays a higher degree of freedom 
and therefore should be considered more complex because it maximizes the possible paths through the existing nodes (and it leads to a higher density of interactions).

\subsection{The energy flux}

The observation of energy efficiency evolution in the economic context tells us that, conversely to a spread opinion, the energy efficiency change is prior to the complexity leap. The related energy conversion process has therefore to be considered as an evolutionary factor affecting the energy balance of the system. It seems that the new conversion rate increases the energy flux through the system and, for the sake energy disposal, the system explores new dissipative structures. Under this perspective, the new structure arrangement rising after the efficiency growth - and the following influx of energy, has indeed to be regarded as a new dissipative strategy of the system. This is the reason why complexity leap increased the energy density of the system, although consuming more energy was not the goal of the system. It is here suggested that complexity leap was, in these circumstances, the strategy for the system to keep growing and to disposal of the incremented energy made available by the efficiency change. Such strategy, however, does not come out of nothing. It represents a particular disposition and organization of systems components previously available, yet not economical. When boundary conditions change such that more economical paths are not any longer exploitable, and if there is an energy inflow such to pose a constant pressure upon the system, the system will thereby explore new settings to dissipate such energy flow.

\section{AN EVOLUTIONARY PATTERN UNDERLIES THE EFFICIENCY-COMPLEXITY DYNAMICS}

When there are forces within the system that push for energy growth - autocatalytic processes - and when the system reaches a point when growth is hindered by external factors, then complexity change becomes compelling. We are arguing here that the natural tendency of autocatalytic processes, such as free market economy, is indefinite expansion, by enlargement, until they reach a point, such as demand saturation, where linear growth is curbed. At this point, the system develops a way by means of a complexity change - a change in structure - to overcome constraints and to continue growing. This could be accomplished by a new product, a new process or a new productive structure.

Nevertheless, a higher system energy level is not always coupled to a higher complexity of the structure. Energy growth is primal to any change in the system, which can sometime evolve a simple expansion (same structure and bigger) or a complexity leap (new structure). It will be attempted to explain where and how such bifurcation occurs in the next chapter.

We have herein advanced the hypothesis of the existence of a common, recursive pattern in evolutionary systems. This pattern underlies a broad, complex thermodynamic process involving the entire system and arises from forces embedded within the system. This pattern can be depicted as a circular process and described as follows: growth-saturation-complexity leap-growth.

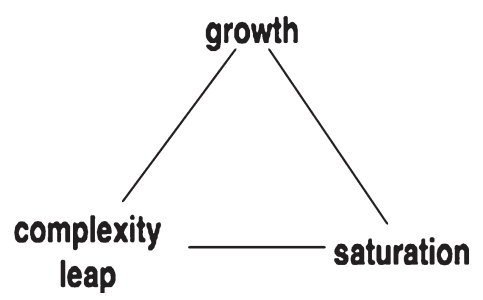




\subsection{Growth-saturation-complexity leap-growth}

This hypothesis was traced by Jevons, in his pioneering contribution to the field of economy, and by Lotka, in biology, although it was not formulated as above. According to Lotka, growth is driven by new species that, thriving on formerly unexploited resources, are able to divert more energy and increase the overall flux through the system. This will happen as long as there is "abundant surplus of energy running to waste" which will constitute substrate ("working substance" in Lotka's words) for life's competition [8]. Growth, in "extent and in number," will go forth and forth until "the working substance or any ingredient of the working substance" reaches a limit. This limit hampers the whole system, as every "subsidiary cycle" within the system is interconnected. This saturation point will stimulate the "subsidiary transformers" (i.e., energy processing components of the system) to "develop new activity, either by acquiring new resources of working substance, or by accelerating its rate of revolution" [8]. Thereafter, the whole system will be further aroused, after having reshaped itself.

In a different context and fairly before Lotka's time, Jevons depicted his own view on economic and social progress as a result of energy availability and provided an insight into this process closely resembling that of Lotka's regarding ecological systems. Indeed, both employ the word "engine" as to refer to the whole system (biological and anthropic) to emphasize the strict interconnection of building components (complexity) and the inner tendency to increase its power. Power is also a key word for both authors. For Lotka, as far as the working substance is copious transformers (species competing for energy), it will tend to maximize the power of the entire system. For Jevons, civilization is the economy of power, meaning that as long as technological progress will make energy (from fossil fuels) more economical, hand labor, animal labor or sun energy (wind and water) will be replaced by mechanical labor. This is for Jevons the "fresh impulse" to growth that will ultimately affect "every branch of manufacture" by prompting them to undertake "works previously not commercially possible" [5]. Yet, "the several branches of industry are closely interdependent, and the progress of any one leads to the progress of nearly all" [5]. This is, according to Jevons, the dynamics of growth as a result of energy efficiency improvement. Nevertheless, growth cannot proceed ad libitum. A "limit," natural or commercial, will surge to hamper growth, because the extent (length of networks or mass of products) or the number of goods cannot be "doubled" indefinitely. A limit, which is not fixed, but an "elastic limit, which may be pushed against a little further, but ever with increasing difficulty. But the new applications of coal are of unlimited character" [5]. Therefore "natural or commercial" limit can be overcome by "new application" of energy or, that is to say, by new products, services, processes, that eventually will increase the interdependence of the industries and upgrade the complexity of the system.

The growth stage therefore relies on the presence of inner forces that drive the system to expand while seeking survival and reproduction. These forces are represented by species - the genome and firms - the capital. Although it is clear how these autocatalytic processes cause the system's expansion, it is less clear how, coupled with efficiency improvements, they can divert more energy into the system or in the words of Lotka, "maximize the energy flow." It must be kept in mind that neither Lotka nor Jevons claims that the overflow of energy is the actual aim of system components. It is rather a result of their interaction with each other and with the environment. Lotka, for example, believes that two main thermodynamic strategies are adopted by organisms to adapt to the environment: maximizing output (power maximum) and minimizing input (efficiency maximum). The former is developed by species thriving in resource abundance and the latter by organisms struggling in scarcity conditions. According to Lotka, by pursuing unexploited free-energy, more energy is driven through the system thus maximizing global output. A case has here been described in which 
the more energy efficient process did not lower system energy level. Energy consumptions grew because the system increased in structural complexity. The hypothesis was thus proposed that this was a specific case underlying a very common thermodynamic pattern in the development of complex systems. This evolutionary pattern was sketched as a circular chain of growth-saturation-complexity leap-growth. According to this view, growth is driven by inner autocatalytic (self-sustaining) processes and nourished by a constant source of free energy. Saturation, a condition of hindering circumtances, is reached by the system because of exogenous causes (spatial, demographical or other factors inherent in the substrate from which growth developed). The complexity leap is a strategy for the system to overcome saturation by rearranging its components into a new structural fashion.

There are somehow similarities between what it is being proposed here and other discussions of the energy dynamic of evolution in ecosystems. As the ecologist Lionel Johnson posed, "Evolution is the outcome of the ultimate ascendancy of the trend toward increasing diversity and acceleration of the energy flow, counteracted and retarded by the individual species attempting to proceed in the direction of greater homogeneity and deceleration of the energy flow" [19]. According to this view, there is an intrinsic tendency in ecosystems to grow in diversity (complexity) that contrasts with internal entropic forces. Here, it is instead suggested that the complexity leap is consequential. The diversity in the ecosystem is the paradoxical result of species' inner tendency of "greater homogeneity" and the limitation of their exponential growth.

Such hindering conditions should not be regarded as merely spatial for they are intended to be any kind of obstacle posed by the environment that affects the growth "in extent and number" of the catalytic process [8]. In other words, the conditions are any limits to the incremental path of the system that eventually compel the system to shift from an "extensive" growth regime to an "intensive" one through a bifurcation point [20].

Biology offers many analogies to the analyzed case of the transport system and productive structure. Most likely, autocatalytic processes preceded the complexity leap and thus, grew in efficiency since the dawn of life on earth. According to many interpretations of evolution, this pattern has been manifest since the early stages of molecular life. In the words of Alberts, the primordial molecules that gave origin to life should have possessed a "crucial property": autocatalysis. In fact, the production of catalysts with a "self-promoting" property would have been favored by natural selection. This special kind of molecules would divert more substrate to their production and would gradually develop through stages of more complex chemical systems. This autocatalytic systems would grow and decay toward chemical equilibrium [9]. Eventually, such "fluctuations" would find a way out of "thermodynamic equilibrium" and persist by indefinitely reinforcing themselves.

It is interesting that under the general assumptions of free energy and raw material availability together with the selective conceptual framework, the crucial role of the efficiency evolution in this pathway is widely recognized. For example, polynucleotides were probably the first form of selfreplicating polymers and were thus capable of replicating themselves and catalyzing their own synthesis. It is remarkable the way enzymes improve efficiency by speeding up the rate of chemical reactions and thus, to a certain extent, by increasing the power of the process. The first big leap in the complexity of life history that gave birth to cellular systems might therefore be attributed to an increase in energy efficiency. The evolution of metabolic reactions is beyond the second big leap in structural complexity, the appearance of multicellular organisms. Glycolysis and in general, any chemical process involving the sugar phosphate's breakdown are the basis of energy conversion in most living systems. It is, however, with the advent of aerobic oxidation that this process can oxidize carbon at the lowest oxidative level and exploit all the free energy embodied in sugars. Respiration once again demonstrates a dramatic increase in power followed by greater complexity. At higher 
levels of organization, efficiency improvements become more subtle, but in the long run, they result in the same outcome. For example, the succession of ecological stages exhibits the same trend in energy efficiency-complexity interaction. (Schneider and Kay demonstrated how the rate of energy captured by ecosystems, the energy thermally radiated in the infrared wavelength over the rate of energy reflected, increases with the complexity of the ecosystem [20].)

Although all ecosystem species rely on the same chemical metabolism, variation in efficiency concerns the fitness of species and the overall effect they produce on the ecosystem's resource cycles. The competition for available resources results in fitter species and the net effect, as cleverly described by Lotka, is an increase in the system's rate of resource and energy output. According to Lotka's categories, the transformer, the biological energy converting process from a thermodynamic perspective, evolves its ability to feed on the substrate by developing strategies, the correlating apparatus, suitable to seek conditions in the external world favorable to its existence. The more advanced correlating apparatus will then rapidly transfer resources to the benefit of the transformer, the success of which will increase the overall energy level (density) of the ecosystem. The role of the apparatus in multiplying "collisions" between transformers and resources interestingly resembles the function of enzymes in increasing the efficiency of chemical reactions in living systems. The enzyme serves to increase the local concentration of substrate molecules at the catalytic site and to hold all of the appropriate atoms in the correct orientation for the following reactions.

In nature, energy efficiency therefore developed throughout biological evolution and permitted the onset of more complex structures. Furthermore, as previously emphasized in this work, an efficiency enhancement entails a power increase for an autocatalytic system. The efficiency evolution, coupled with autocatalytic processes, was thus the main source of a growing incoming energy flux in biological systems (and anthropic systems as well). The growing energy density and the growing energy efficiency are commonly connected to a higher complexity system. This latter feature is generally considered the cause of the first two. It is argued here that the very contrary is true. System complexity grows after the growth of energy density and efficiency and because of the increasing energy flow generated by the efficiency enhancement. The efficiency improvement is the trigger of the structural change and the complexity growth as in the case study of trucks and the productive structure object of this work. (It is also a third condition that regards compelling system boundaries.)

\section{COMPLEXITY GROWTH, DEGREE OF FREEDOM AND SPATIAL GRADIENTS}

We would now like to highlight that the kind of complexity growth expressed by biological processes is similar to the one we formerly depicted for the economic structure. This is indeed the case if we apply the paradigms that we used to analyze structural complexity growth. For example, the food degradation process occurs at two levels of organization: in cells, specifically in mitochondria and in the digestive apparatus. This process entails two levels of catabolic or metabolic rates and thus, two control systems, one at a lower, cellular level and the other at a higher, bodily level. The shift from unicellular to multicellular organisms was thus a complexity leap by the homeostatic system theory. The scale effect is also easily explained and it concerns, for example, ecosystem's size. It is well known that when ecosystems shrink below a certain level, biodiversity decreases as well [21]. Complexity also increases when the system can rearrange its components to increase the number and the path length or the speed of interactions. An example in cells is represented by the internal skeleton of microtubules that increase the speed of molecules across the cell by means of diffusion. In ecosystems, there are food chains and predator-prey dynamics. The four paradigms, indeed, express a unique view of complexity based on the concept of geometry. They all share the idea of a molten structure that evolves configuration by rearranging itself into a new form or a higher 
scale of aggregation in an effort to explore new shapes. The concept of geometry was also one of Lotka's key points. He was firmly convinced that this was a peculiar feature of thermodynamic of living systems and thus, of a sort completely different from those normally addressed by physics. Whereas the latter mainly dealt with "structureless systems," of the like of chemical coefficients, the former must approach "geometrical features" [8]. Complex systems display a spatial gradient, which is sometimes many orders of magnitude larger than gradients on a molecular scale [17] and are therefore of little use to understand cell organization and of even less use to understand body metabolism or population dynamics. Is such little use avoidable for the sake of evolutionary theory in the complex systems field? The bulk of Lotka's theory was based on the belief that both biology and thermodynamics advance by breeding with each other in a sense and thus, by mutual contamination. Thermodynamics would improve itself and expand its ability, although well founded and uncontroversial, of defining what "was not possible" in all the vast circumstances of energy conversion. Biology, however, would dismiss altogether the misplaced, long enduring, belief that nature is a mere sum of parts and life, a phenomenon intrinsically diverse from inanimate matter. In Lotka's opinion, science managed to progress due to the merging of these two fields, forming the basis of a new paradigm: "What is needed, in brief, is something of the nature of what has been termed 'Allgemeine Zustandslehre', a general method or Theory of States" [8]. This new, forthcoming theory would deal with biological statistics, like thermodynamics accept problems in terms of physical data [8]. Lotka paved the way for the onset of a new methodology, the shape of which is still undergoing a constant transformation. He found many supporters of his idea that power maximization is a result of selection, chance, and the inflow of free energy. His "power principle," which he never actually formulated as a principle, has been restated in many ways in different scientific fields. His model of population dynamics based on a simple system of differential equations became even more famous. However, among all of Lotka's suggestions and intuitions, the one envisaged in the sentence above, the quest for a "theory of states" in life sciences, is the most neglected. A possible explanation for this disregard could be the difficulties of computing or even defining a set of states in the field of life sciences. (Thermodynamic principles are explainable in terms of energy microstates of matter. In this context, entropy reflects the number of different ways microstates can be combined to give a particular macrostate: the larger the number of microstates for a given macrostate, the larger the entropy. The macrostate with the largest number of microstates is thus the most likely to exhibit probabilistic behavior. The tendency of a system to increase its entropy is thereby explained.)

5.1 The complexity leap: new states with higher degrees of freedom, achieved by pursuing new spatial gradients

This is indeed a very difficult, perhaps even impossible, achievement: defining all possible states for evolutionary systems. A golden rule of statistics states that to compute all probable distributions, a system needs to be stationary. System states therefore need to be previously known or deducible from present states. Evolutionary systems, however, are not stationary or at least not on an evolutionary time scale. Nevertheless, if complexity growth must be regarded as the emergence of completely new and previously unavailable states, it is possible to detect past occurrences and conditions.

Recently, Fath gave a full survey of ecological goal functions and showed that, although seemingly incoherent, they are congruous when considering the three fundamental properties: maximize input and direct flows; maximize retention time; maximize cycling [7]. According to Fath, these goals or orientors are mutually consistent and interdependent of fulfillment. All the 10 evaluated goal functions, in light of these orientors, depict the coherent behavior of ecological systems. Fath 
also emphasizes that the network structure is the arch-key that makes this possible [7]. The theoretical framework is very consistent with what has been contended thus far regarding the evolution in complex systems. Nevertheless, it is still implicitly teleological. The maximization of energy flux rather than a mere result of autocatalytic forces is presented as a goal. The network structure development that eventually results in complexity growth at any system level is otherwise the outcome of such forces in the context of hindering boundary conditions. It is the simple growth and thus, the normal behavior in the absence of such conditions. The system, driven by autocatalytic processes, develops in a primary and spatial manner as well as in a secondary and geometrical (structural) one. It is such geometrical development that enables the system to increase its degree of freedom and to host more energy (or energy density rate) within the same constraints. When this complexity change emerges, the incoming structure previously available to system components becomes more probable. The boundary conditions ultimately determine the likelihood of the new structure. It is also true for simple physical systems that changing a system's boundary conditions alters its degree of freedom. For example, if the pressure of a gas increases, its degree of freedom decreases and it consequently becomes a liquid. At the same time, when a liquid changes its motion regime, as in Bénard cells, from a pure, random dissipative system to a global dissipative one, which displays features several magnitudes larger than molecules, a superstructure arises that was previously available yet very unlikely or even impossible. Gravity and viscosity constraints make such a structure, beyond a certain level of energy (heat) input, possible. The random motion of molecules reflects one gradient, the temperature, which is not spatial (geometrical), while the superstructure is exposed to the spatial gradient. That is to say, while the first gradient is defined by one variable, the latter is described by three variables and probabilities consequently change. Dissipation onto one variable is therefore more probable than onto three variables, unless boundary conditions render the former impossible. In Bénard cells, such conditions are exemplified in Van der Waals forces, the low heat capacity of water, and restrained vessel thickness [17, 22, 23].

The connectivity recasts the same trade-off in a network system's conceptual framework. A network system grows in complication as long as a new component is connected on the same hierarchical level and it grows in complexity when a new component is introduced on a higher hierarchy [24]. The emergence of a new hierarchy entails coherent behavior for lower level components to the same extent as molecules in Bénard cells and, most importantly, the onset of a new spatial gradient for the higher component, which must now recognize system boundaries. (It is noteworthy that, from a cybernetic point of view, degree of freedom (number of independent variables) and gradient (vector of derivatives of the dependent variable on the independent variables) are both correlated to the introduction of new variables in the system where the amount of components are held constant. This notion indeed constitutes an obstacle to the attempt of modeling evolution.) On a molecular scale, cells in the body behave like a network. From the standpoint of the organism, however, they act as a whole unit. It is indeed true that "free" cells in substrates are mainly exposed to chemical gradients (temperature, pressure and gravitational gradients as well), while "embedded" cells in tissues that form organs are described by spatial, three dimensional, gradients. (Cell division in developmental stages follows essentially a spatial gradient, like those regulated by homeotic genes.)

The present case study, the dynamic interplay of energy efficiency in the transport sector and productive structure, displays the same evolutionary pattern of structural change to a system of greater degrees of freedom. This pattern also seems to exhibit the same trend toward higher system gradients. The system, to change its degree of freedom, explores new gradients previously inaccessible to its components. Strong analogies embrace economy and we thus contend that complexity growth can be approached from a thermodynamic perspective accordingly in the economic field. The extent to which the shift from a fordian to a post-fordian productive structure increases the 
degree of freedom of the productive system was formerly discussed, where the transport sector is included in the domain of the productive system. The increased hierarchy, the emergence of national markets and consequently, higher specialization and, above all, the emergence of a network-shaped, wide productive chain in place of a star-shaped one, highlight the incremented degree of freedom of the system. Such higher degrees of freedom were obtained by the system's search for new and more favorable economic conditions for production, such as different labor, stocking, and supplying costs. For example, after globalization, firms could explore labor costs according to various national legislations and average incomes. The same occurred for financial and fiscal conditions or the proximity to productive districts. All the factors became variables for the firms to optimize by allocating, fiscally or economically, part of the production (or capital) chain to several countries. What was predominately about negotiation with policy makers, labor unions or banks, became a matter of logistic and transport costs. Firms could thereby reduce production costs by selecting where to set plantations or rely on supplies. It is in this sense that globalization produced the rise of new spatial gradients in the productive system.

\subsection{Spatial symmetry rupture}

The statement about the system searching for new gradients may appear teleologically anew. Teleology was previously discharged on the ground of the evolutionary path, to be now recast in the form of a sentient agent, the system, looking for gradients. It is indeed a misrepresentation of the actual process, which occurs probably because of changing boundary conditions, and is due, above all, to semantic conciseness. As a matter of fact, the rise of new gradients concerns the space wherein the system develops itself. A new gradient springs out of a spatial symmetry rupture. The symmetry rupture is due to two counteractive forces: system's components are mutually exposed to some hindering forces (viscosity and gravity, in the case of Bénard cells), formerly negligible or absent, and an increasing energy flux. Symmetry in space can thus be re-established when such forces cease or are surmounted (temperature rises over a certain threshold in the case of Bénard cells).

It is an external force that, on the one hand, reduces the degree of freedom of single particles and on the other, by introducing a new gradient, collectively enables the system to augment its degree of freedom by achieving new forms of organization. Degrees of freedom of particles and system are thus inversely related, as determined by the "symmetry rupture."

It is noteworthy that laws of mechanics are based on the homogeneity and isotropy of space. The random motion of gas molecules is thus described, microscopically, by kinematics because gravitational force is negligible and symmetry in space is maintained. In the case of liquids, gravitation counts and rotational symmetry is broken. Complexity grows by succeeding symmetry fractures. In the case of the productive structure's evolution, it is manifest that space was isotropic in the former state (graph A, Fig. 3) and non-isotropic in the latter (graph B, Fig. 3): a spatial symmetry breaking occurred. What made this spatial gradient rise was, initially, a reduction in firms' degrees of freedom in production setting, brought about by a new, more rigid labor market.

\section{CONCLUSION}

We are suggesting here that a new "theory of states" for complex systems should depart form the above-mentioned hints of evolutionary pattern. There is strong evidence in several fields of recursiveness with regard to the dynamic, growth-saturation-complexity leap-growth. Moreover, there is also evidence supporting complexity growth as an increase in degree of freedom by furthering spatial gradients. However, the acknowledgment of the key role of energy as an evolutionary factor does not negate the importance and specificity of scientific and social disciplines. We are 
otherwise seeking, provided the wide range of analogies among complex systems of various genres, more general explanations for similar phenomena. Such explanations should overcome, in terminology and in categories, single disciplinary approaches, by maintaining their own validity in their specific domains. Further research should address the need to formalize the change in degree of freedom of a system's structure brought about by new gradients. That is, additional research should calculate probabilities in system macro states based on the change in microstates created by evolutionary processes.

Finally, given that energy efficiency improvements generally introduce more energy into the system, we analyzed the recursive evolutionary pattern, from ecological systems to economies, that drives complex systems to change their structure and grow in complexity. It was proposed that such a pattern could be summarized in a circular loop involving three stages: growth, saturation, complexity leap. Energy efficiency improvements came about as a result of growth hindering conditions that eventually feedback to energy converting processes in an attempt to overcome steady state. The system grows by inner self-reinforcing (self-sustaining and reproducing) processes, such as autocatalytic reactions in the field of life or the capital cycle in the field of economy. The interplay of these immanent exponential dynamics with energy efficiency improvements diverts more energy into the system. The increased energy inflow is thereby dissipated by augmenting system components in extent (expansion or growth in complication) at the first stage. When expansion is restricted by internal (substrate or amount of resources and factors) or external (spatial) constraints, the system exploits the complexity-growth strategy to dissipate the incoming and increasing energy flux. The complexity growth is a structural rearrangement of system components to increase their degree of freedom. A higher degree of freedom entails a new gradient available to system components. In a feedback process, higher degrees of freedom are attained by exploring new gradients previously less "economical" (and less likely, from a probabilistic standpoint) and made less energy costly by efficiency improvements. On a broader standpoint, a new gradient is imposed to the system by two counteractive forces: the inner tendency to expand and constrains posed by saturation. The gradient that was previously available, nevertheless, was irrelevant for the scale of single components (because of their higher degree of freedom). When saturation is reached, constrains reduce degrees of freedom of single components and therefore space, where components move, is exposed to a symmetry rupture. When symmetry is broken, a favored orientation for interactions is introduced and, despite single components' degrees of freedom being reduced, the frequency and the speed of overall interactions augment the degrees of freedom of the whole system. This is what we intend for a "complexity leap."

\section{REFERENCES}

[1] Binswanger, M., Technological progress and sustainable development: what about the rebound effect? Ecological Economics, 36, pp. 119-132, 2001.

[2] Geening, L.A., Greene, D.L. \& Difiglio, C., Energy efficiency and consumption - the rebound effect - a survey. Energy Policy, 28(6/7), pp. 389-401, 2000.

[3] Blake, A., Jevons' paradox. Surveys. Ecological Economics, 54, pp. 9-21, 2005.

[4] Khazzoom, J.D., Economic implications of mandated efficiency in standards for household appliances. The Energy Journal, 1(4), pp. 21-40, 1980.

[5] Jevons, W.S., The Coal Question - An Inquiry Concerning the Progress of the Nation, and the Probable Exhaustion of Our Coal-Mines, Augustus M. Kelley: New York, 1965.

[6] Odum, E.P., Ecology: A Bridge between Science and Society, Sinauer Associates, Inc.: Sunderland, MA, 1997.

[7] Fath, B., Patten, B. \& Choi, J., Complementary of ecological goal functions. Journal of Theoretical Biology, 208, pp. 493-506, 2001. 
[8] Lotka, A., Elements of Mathematical Biology, Dover Publications, Inc.: New York, 1956 (first publication: Elements of Physical Biology, Williams and Wilkins Co., Inc., 1924).

[9] Alberts, B., Bray, D., Lewis, J., Raff, M., Roberts, K. \& Watson, J.D., Molecular Biology of the Cell, Garland Publishing, Inc.: New York, 1994.

[10] Chaisson, E., Cosmic Evolution - The Rise of Complexity in Nature, Harvard University Press: Cambridge, MA, 2001.

[11] Lipietz, A., Towards a New Economic Order, Posfordism, Ecology and Democracy, Polity Press: Cambridge, UK, 1992.

[12] Tuttotrasporti, Editoriale Domus S.p.A., Rozzano, Milan, Italy.

[13] Krugman, P., Cooper, R.N. \& Srinivasan, T.N., Growing world trade: causes and consequences. Brookings Papers on Economic Activity, 1995(1), 25th Anniversary Issue, pp. 327-377, 1995.

[14] OECD, Economic regionalisation and intra-industry trade: Pacific-Asian perspectives, OECD Development Centre, Working Paper No. 53, 1992.

[15] OECD, Intra-industry and intra-firm trade and the internationalisation of production, OECD, Economic Outlook 71, Part VI, Paris, 2002.

[16] OECD, Liberalisation and structural Reform in the freight transport sector in europe, OECD, Paris, 1997.

[17] Prigogine, I. \& Stengers, I., Order Out of Chaos, New Science Library: Boulder, CO, 1984.

[18] El-Samad, H., Prajna, S., Papachristodoulou, A., Doyle, J. \& Khammash, M., Advanced methods and algorithms for biological networks analysis. Proceedings of the IEEE, 94(4), pp. 832-853, 2006. Postprint available free at: http://repositories.cdlib.org/postprints/1474.

[19] Johnson, L., Thermodynamic origin of ecosystems. Entropy, Information, and Evolution, eds B.H. Weber, D.J. Depew \& J.D. Smith, MIT Press: Cambridge, MA, 1988.

[20] Schneider, E.D. \& Kay, J.J., Life as a manifestation of the second law of thermodynamics. Math. Comp. Model, 19(6-8), pp. 25-48, 1994.

[21] Loehle, C., Challenges of ecological complexity. Ecological Complexity, 1, pp. 3-6, 2004.

[22] Swenson, R., Autokatakinetics, evolution, and the law of maximum entropy production: a principled foundation toward the study of human ecology. Advances in Human Ecology, ed. L. Freese, JAI Press: Greewich, CT, pp. 1-47, 1989.

[23] Swenson, R., Emergent attractors and the law of maximum entropy production: foundations to a theory of general evolution. Systems Research, 6, 187-197, 1989.

[24] Allen, T.H.F. \& Starr, T.B., Hierarchy: Perspectives for Ecological Complexity, University of Chicago Press: Chicago, IL, 1982. 